\title{
3D modelling of the flow of self-compacting concrete with or without steel fibres. Part II: L-box test and the assessment of fibre reorientation during the flow
}

\author{
R. Deeb · S. Kulasegaram • B. L. Karihaloo
}

Received: 12 December 2013 / Revised: 10 February 2014 / Accepted: 19 March 2014 / Published online: 9 April 2014

(C) Springer International Publishing Switzerland 2014

\begin{abstract}
The three-dimensional Lagrangian particlebased smooth particle hydrodynamics method described in Part I of this two-part paper is used to simulate the flow of self-compacting concrete (SCC) with and without steel fibres in the L-box configuration. As in Part I, the simulation of the SCC mixes without fibres emphasises the distribution of large aggregate particles of different sizes throughout the flow, whereas the simulation of high strength SCC mixes which contain steel fibres is focused on the distribution of fibres and their orientation during the flow. The capabilities of this methodology are validated by comparing the simulation results with the L-box test carried out in the laboratory. A simple method is developed to assess the reorientation and distribution of short steel fibres in selfcompacting concrete mixes during the flow. The reorientation of the fibres during the flow is used to estimate the fibre orientation factor (FOF) in a cross section perpendicular to the principal direction of flow. This estimation procedure involves the number of fibres cut by the section and their inclination to the cutting plane. This is useful to determine the FOF in practical image analysis on cut sections.
\end{abstract}

Keywords 3D simulation - Self-compacting concrete . L-box, Smooth particle hydrodynamic (SPH) .

Short steel fibres · Fibre orientation

R. Deeb · S. Kulasegaram · B. L. Karihaloo ( $\varangle)$

School of Engineering Cardiff University,

Cardiff CF24 3AA, UK

e-mail: KarihalooB@Cardiff.ac.uk

\section{Introduction}

The corrected incompressible mesh-less Lagrangian SPH method described in Part I of this two-part paper is implemented to simulate the filling and passing ability in a confined zone in the L-box. The flow-ability of SCC mixes with and without fibres has been assessed using the slump cone test in Part I of this paper [1]. Other important properties of an SCC mix in a free surface flow besides its flow-ability are the filling and passing ability in a confined zone. The filling ability, the blocking resistance and the segregation resistance are investigated in the L-box test. For that reason, 3-dimensional simulations of the SCC mixes were undertaken in the L-box configuration to examine the flow and filling characteristics of the SCC mix in a restricted region and its ability to pass through gaps between steel bars without any segregation or blockage of its larger components. The simulation results are compared with the laboratory tests.

As in Part I, the 3D simulation of the SCC mixes without fibres will emphasise the distribution of large aggregate particles of different sizes throughout the flow. On the other hand, the simulation of high strength SCC mixes (compressive strengths in the range 100-160 MPa) which contain between 0.5 and $2.5 \%$ by volume steel fibres will focus on the distribution of fibres and their orientation during the flow. The capabilities of this simulation methodology will be validated by comparing the simulation results with the L-box tests carried out in the laboratory.

The orientation and the distribution of the short steel fibres will be monitored throughout the flow and the change in orientation will be statistically analyzed. It will be shown that the fibres tend to align themselves with the principal direction of flow but remain mostly randomly distributed perpendicular to this direction. The reorientation of the fibres during the flow will be used to estimate the fibre orientation fac- 
Table 1 The mix constituents per $\mathrm{m}^{3}$ of Mix 1, Mix 2, Mix 3 and Mix 4

\begin{tabular}{|c|c|c|c|c|}
\hline Constituents & Mix 1 & Mix 2 & Mix 3 & $\operatorname{Mix} 4$ \\
\hline Cement (kg) & 500 & 500 & 543.5 & 543.5 \\
\hline Micro-silica (kg) & 75 & 75 & 214 & 214 \\
\hline Ground granulated blast furnace slag (GGBS) (kg) & - & - & 311.5 & 311.5 \\
\hline Limestone powder $<2 \mathrm{~mm}$ & 200 & 200 & - & - \\
\hline Coarse aggregates $(\mathrm{kg})$ (crushed limestone) $<10 \mathrm{~mm}$ & 833 & 833 & - & - \\
\hline Sand $<2 \mathrm{~mm}$ & 700 & 700 & - & - \\
\hline \multicolumn{5}{|l|}{ Quartz Sand (kg) } \\
\hline $9-300 \mu \mathrm{m}$ & - & - & 470 & 470 \\
\hline $250-600 \mu \mathrm{m}$ & - & - & 470 & 470 \\
\hline Water $(\mathrm{kg})$ & 138 & 138 & 188 & 188 \\
\hline Fibres (30 mm long with crimped ends, volume fraction) & - & $0.5 \%$ & - & $2.5 \%$ \\
\hline Super-plasticiser/water & 0.14 & 0.14 & 0.28 & 0.28 \\
\hline Water/(cement+micro-silica+GGBS) & 0.24 & 0.24 & 0.18 & 0.18 \\
\hline Flow spread in cone test $(\mathrm{mm})$ & 805 & 760 & 910 & 850 \\
\hline$t_{500}$ in slump test (s) & 2.2 & 3 & 3 & 3 \\
\hline$t_{200}$ in L-box (s) & 0.35 & 1.88 & 0.3 & 1.9 \\
\hline$t_{400}$ in L-box (s) & 0.76 & 5.1 & 0.75 & 5.6 \\
\hline Level-off time in L-box (s) & 7.23 & 38 & 0.95 & 54 \\
\hline Plastic viscosity (Pa s) & 9.77 & 42.1 & 3.1 & 54.3 \\
\hline Compressive strength (MPa) & 80 & 100 & 140 & 160 \\
\hline
\end{tabular}

tor $(\mathrm{FOF})$ in a cross section perpendicular to the principal direction of flow.

\section{Development of mixes}

In order to test the ability of the SCC mixes, described in Part I of this paper, to fill the formwork containing reinforcement under its own weight, the L-box apparatus is used. The vertical leg of the L-box is filled with the SCC mix. At the bottom of this leg is a gate with two or three rods in front of it. When the gate is lifted, the mix flows into the horizontal part of the L-box through the gaps between the rods. The times for the mix to reach $200\left(\mathrm{t}_{200}\right)$ and $400 \mathrm{~mm}\left(\mathrm{t}_{400}\right)$ from the gate are recorded, as well as the time it takes the mix to level off in the horizontal leg of the L-box. Again, it is required that no large aggregate particles or fibres be blocked by the rods. These times are given in Table 1 for the four mixes described in Part I. The filling ability in L-box may be judged from Figs. 1 and 2.

\section{Initial Configuration and Boundary Conditions}

As mentioned in Part I, appropriate initial and boundary conditions need to be applied when solving the mass and momentum conservation equations. In the simulation of the L-box test, the SCC mix in the vertical leg of the box is at rest until the gate is opened. As far as the boundary conditions are concerned, three types of boundary conditions need to be considered; a zero pressure condition on the free surface, Dirichlet boundary condition at the walls of the box, and Neumann conditions on the pressure gradient (this additional zero pressure gradient condition is needed only for solving the secondorder pressure Poisson equation), as illustrated in Fig. 3.

Four arrays of rigid dummy particles placed outside the wall and the base of the L-box were used to implement the boundary conditions. Dirichlet and Neumann boundary conditions were imposed on the wall and the base of the Lbox. Sliding frictional resistance between the SCC mix and rigid boundaries was imposed as illustrated in Fig. 3 with a dynamic coefficient of friction between the SCC mix and steel equal to $0.55 \mathrm{Ns} / \mathrm{m}$. This coefficient has been estimated previously (see Part I) [1] by matching the $t_{500}$ in a slump cone test with the simulated result. As the L-box gate is lifted, the condition $P=0$ is imposed on the newly formed free surfaces of the flowing SCC mix.

\section{Treatment of aggregates}

As in the simulation of the slump cone test in Part I, the number of particles used to represent the volume of the L-box contents sets a lower limit on the volume element 

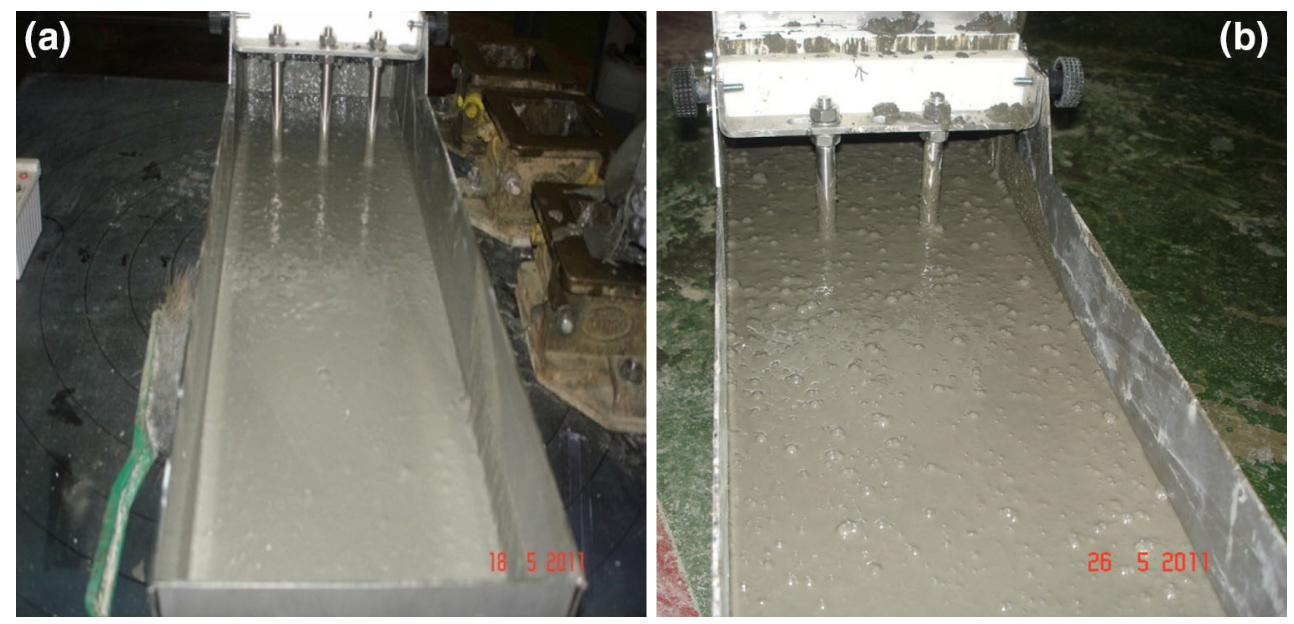

Fig. 1 Filling and passing ability of SCC Mix 1 (a) and Mix 2 (b)
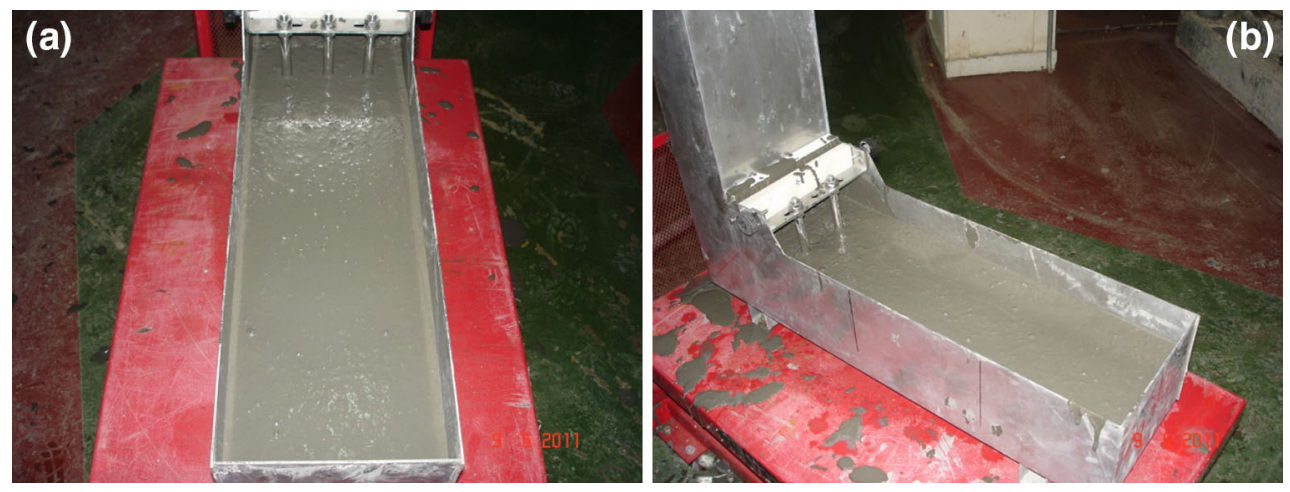

Fig. 2 Filling and passing ability of SCC Mix 3 (a) and Mix 4 (b)

that can be distinguished from the homogeneous mass, i.e. the resolution of the modelling technique.

In the 3D simulations of the L-box, a total of 59568 particles have been used to represent the volume of the mix (129 $x 10^{5} \mathrm{~mm}^{3}$ ) giving a resolution of $216.56 \mathrm{~mm}^{3}$ if all particles have the same density as the viscous continuum. The actual resolution may be somewhat different if the particles have different densities (see Table 2 below). Thus, in Mix 1 (Table 1) the large aggregates that can be distinguished from the homogeneous mass must have a volume exceeding this minimum. It is for this reason that only the aggregates of size $8 \mathrm{~mm}$ and above have been chosen.

\section{Treatment of Fibres}

The methodology based on SPH used here to monitor the fibres during the flow proposed by Kulasegaram and Karihaloo [2] was described in Part I.

Note however that because of the presence of sharp corners, it may be necessary during the flow around a sharp corner to relax temporarily the condition on the constant distance between fibre end particles until they have rounded the corner.

\section{Simulation results}

\subsection{L-box test results for SCC mixes without fibres}

Simulations of the a typical SCC mix (Mix 1, Table 1) were undertaken in the L-box configuration to examine the flow and filling characteristics of the SCC mix in a confined volume and its ability to pass through 3 steel bars without any segregation of its large components. The gap between the neighbouring bars is $41 \mathrm{~mm}$. The simulation results were also compared with the laboratory tests. In the 3D simulations the volume of the mix in the L-box was simulated by 59568 particles. Two simulations were performed; one in which the aggregates equal to or larger than $8 \mathrm{~mm}$ in size were treated separately from the viscous paste, and the second in which the entire volume of mix in the L-box was treated as homogeneous. The volume fractions of aggregates larger than or 

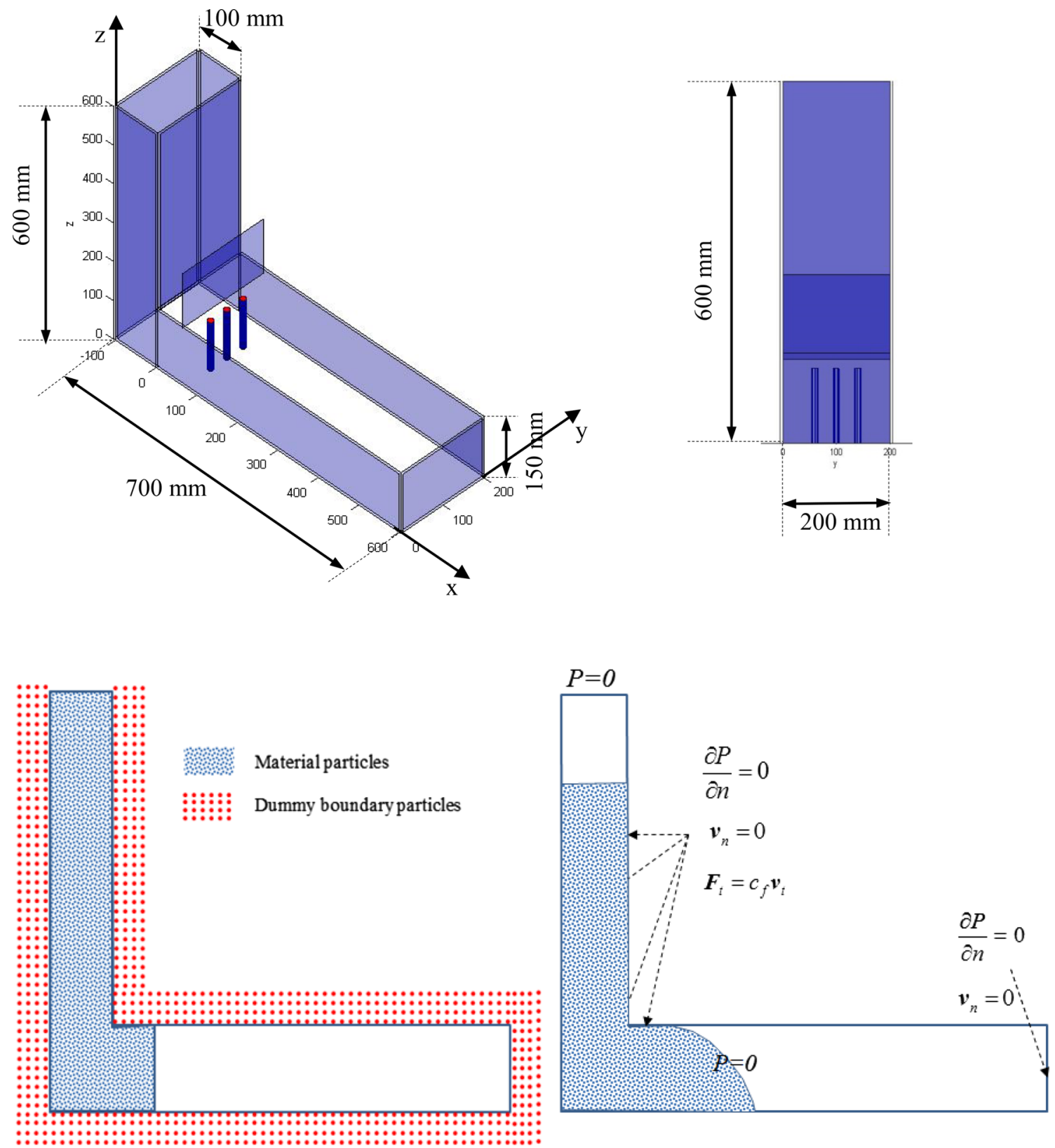

Fig. 3 L-box test boundary conditions ( $P$ - pressure, $v_{n}$ - normal velocity, $v_{t}$ - tangential velocity, and $c_{f}$ - the dynamic coefficient of friction). Pressure vanishes on a free surface. Note that the condition that the

equal to $8 \mathrm{~mm}$ in Mix 1 and the number of particles representing them in the 3D L-box test simulation are reported in Table 2. The results of these simulations are illustrated in Figs. 4 and 7 at various time steps.

As can be observed from the simulation results illustrated in Fig. 4, the larger aggregates stay homogenously distributed at all times during the flow exactly as in the L-box test in the laboratory. The times needed for the mix to reach 200 and $400 \mathrm{~mm}$ were 0.3 and $0.64 \mathrm{~s}$, respectively. Furthermore, the ratio of the concrete height at the end of the horizontal normal pressure gradient vanishes on rigid surfaces is only needed in the solution of the pressure Poisson Equation (10) in [1]

section to the height of the remaining concrete in the vertical section is more than the minimum recommended value 0.8 [3]; these results correlate very well with the experimental results (Table 1) $[14,15]$. By cutting the L-box by vertical planes (A, B and C as illustrated in Fig. 4(d)) after 6 s flow time, the statistics of the large aggregates $(\geq 8 \mathrm{~mm})$ along these sections can be investigated to check whether or not they stay homogeneously distributed in the mix.

Figure 5 shows how the aggregates are distributed in the vertical cross-sections after 6 s flow time with no visible 
Table 2 Volume fractions of aggregates larger than or equal to $8 \mathrm{~mm}$ in Mix 1 and the number of particles $\left(\mathrm{N}_{p}\right)$ representing them in the 3D simulation of the L-box test and their assigned volume $\left(\mathrm{V}_{a}\right)$

\begin{tabular}{llllll}
\hline SCHPC (Mix 4) & Particle diameter $(\mathrm{mm})$ & Density $\left(\mathrm{kg} / \mathrm{m}^{3}\right)$ & Volume fraction $(\%)$ & $\frac{3}{3 \text { D L-box test }}$ \\
\cline { 4 - 6 } & & & $\mathrm{N}_{p}$ & $\mathrm{~V}_{a} /{\mathrm{particle}\left(\mathrm{mm}^{3}\right)}$ \\
\hline Particles $<8 \mathrm{~mm}$ & $<8 \mathrm{~mm}$ & 2332.4 & 74 & 50579 & 179.38 \\
Aggregates $\geq 8 \mathrm{~mm}$ & 10 & 2800 & 15 & 3696 & 597.42 \\
& 8 & 2800 & 11 & 5293 & 305.88 \\
& Total & 2454 & 100 & 59568 & - \\
\hline
\end{tabular}

\section{(a)}

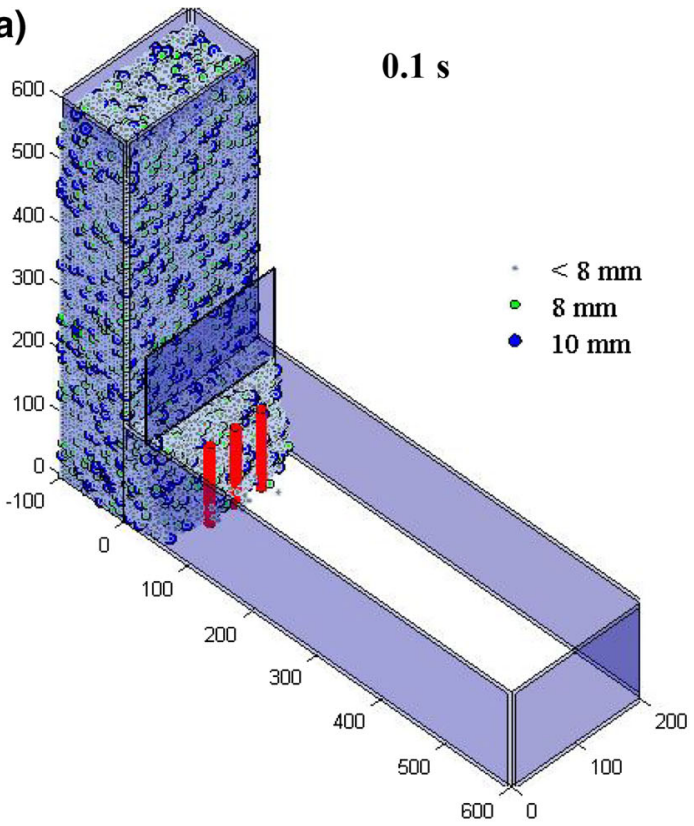

(c)

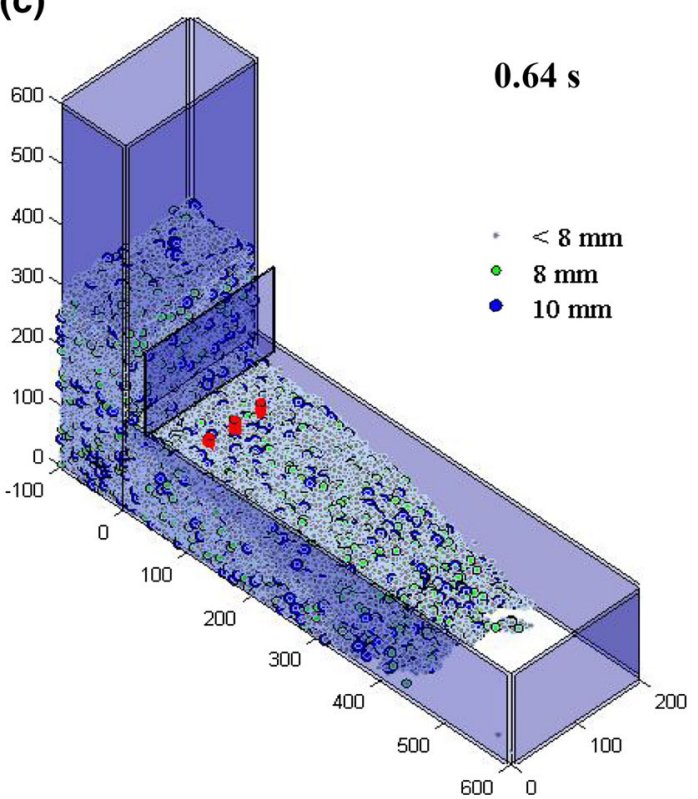

(b)

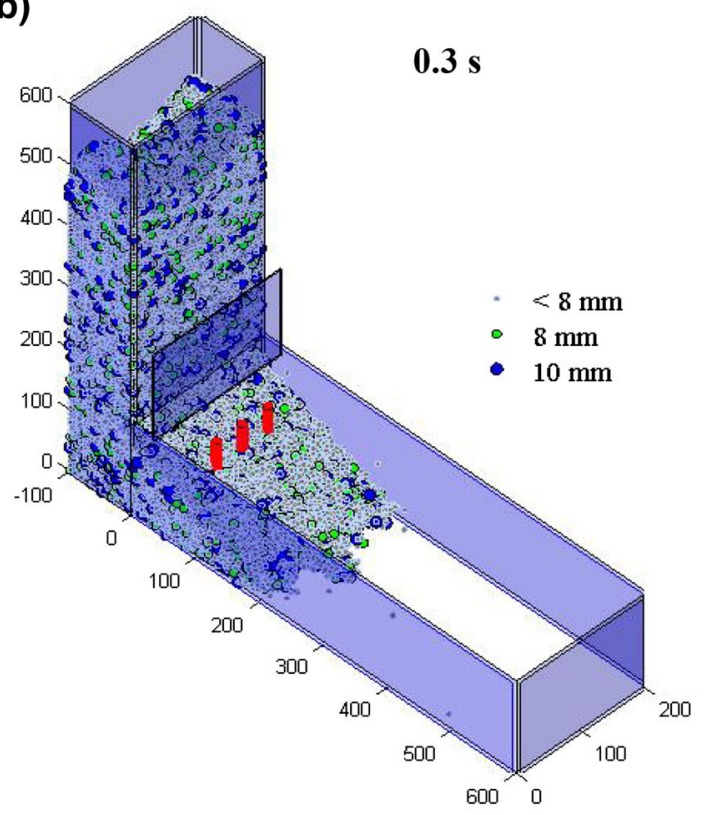

(d)

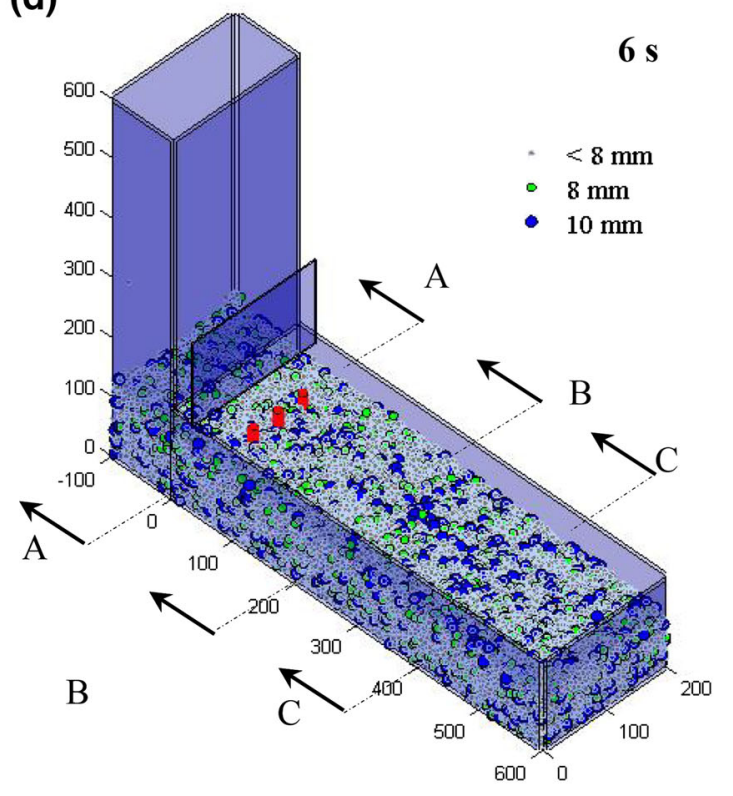

Fig. 4 The flow of SCC Mix 1 in the L-box after $0.1 \mathrm{~s}, 0.3 \mathrm{~s}, 0.64 \mathrm{~s}$, and $6 \mathrm{~s}$ showing the large aggregates 
settlement of the larger aggregates $(\geq 8 \mathrm{~mm})$. Note the decreasing height of the mix along the direction of flow, i.e the height decreases from section A to C.

That the larger aggregates are indeed distributed in an identical manner along these three sections can be ascertained by performing a statistical analysis of these aggregates to estimate the best fit Probability Density Function (PDF) which in this case turns out to be the Weibull distribution function amongst the many distribution functions that were tried. It can be seen from the histograms in Fig. 6 that the larger aggregate distribution is almost identical along the three cross-sections.

This 3-dimensional simulation notably presents an improvement over the 2-dimensional simulation results [4], achieving more realistic flow times of SCC in the horizontal section of the L-box. Table 3 compares the 2D and 3D simulation results of the L-box with the laboratory results.

In the 3D simulations, although the inclusion of the frictional resistance to the flow due to the vertical sides of the horizontal part of the L-box improves the flow times of SCC in the horizontal section of the L-box, and therefore gives more realistic flow times, there is an inevitable delay in the laboratory test in manually lifting the gate to release the mix thus increasing the measured times. The delay is the more, the larger the content of coarse aggregate and/or fibres in the mix.

Figure 7 illustrates the numerical simulations of Mix 1 treated as a homogeneous mass. The distances 200 and 400 $\mathrm{mm}$ were traversed in 0.3 and $0.64 \mathrm{~s}$, respectively, exactly as in the heterogeneous simulation illustrated above (Fig. 4). Moreover, the surface of flow is smooth and looks identical to that observed in the laboratory test on this mix. The simulation results obtained here confirms that Mix 1 is a good self-compacting mix in the sense that it flows as a homogeneous mass, fills all the far corners of the box, and passes through reinforcement bars without any segregation of the heavier particles.

\subsection{L-box results for SCC mixes with fibres}

The SCC mixes with fibres (Mixes 2 and 4 in Table 1), which contain between 0.5 and $2.5 \%$ by volume steel fibres has been modelled using three-dimensional Lagrangian smooth particle hydrodynamics (SPH) in the L-box. These simulations will provide an insight into the distribution of fibres and their orientation during the flow. A two-dimensional simulation of such mixes [4] is rather misleading because all the fibres appear in a single plane. The accurate picture can only be gained by using the three-dimensional flow simulation which shows the actual distribution of fibres and their orientation during the flow.

In these simulations and because of the inclusion of 30 $\mathrm{mm}$ long steel fibres, only two steel bars will be used as
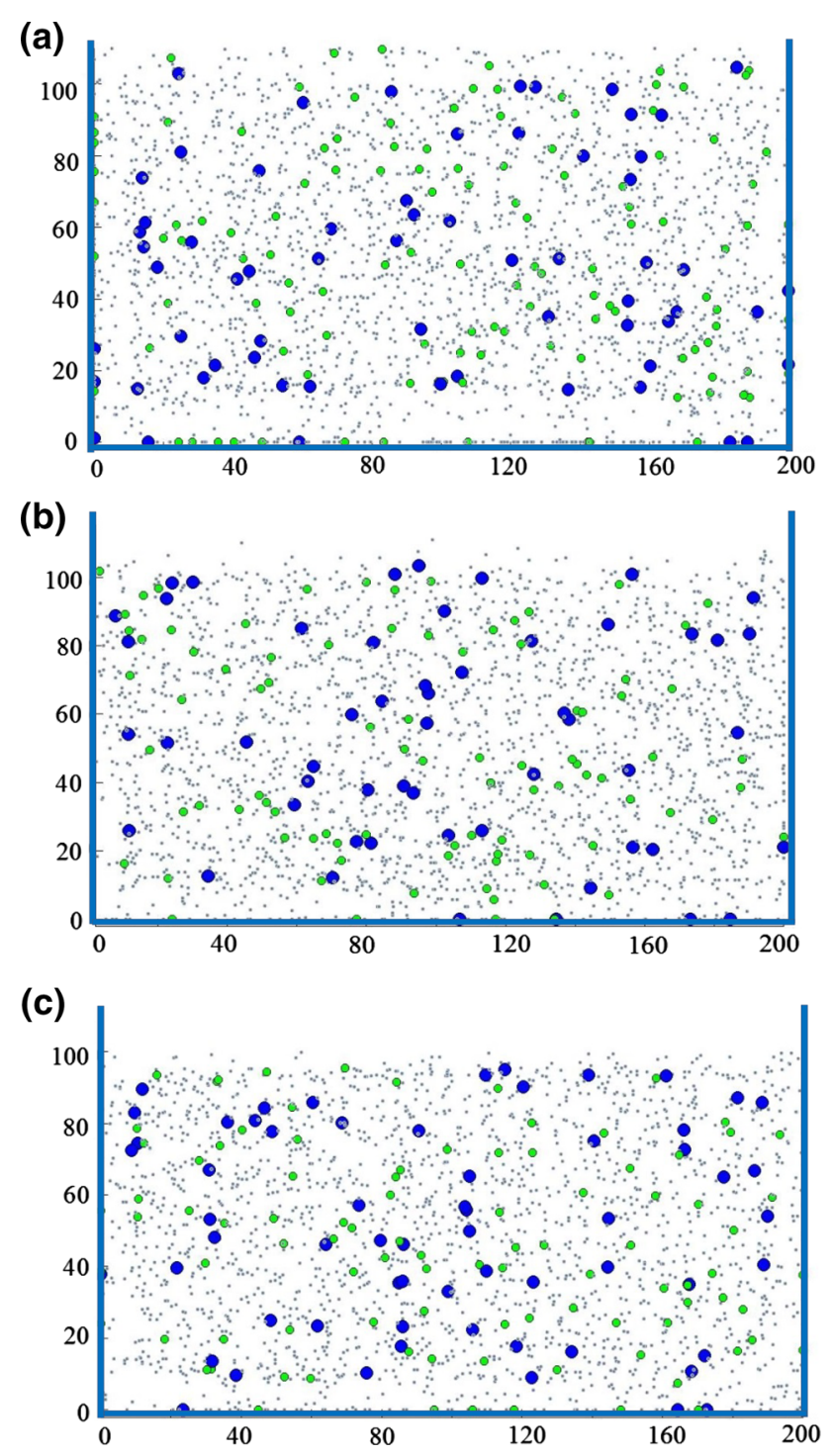

Fig. 5 Vertical cross-sections (a, $\mathbf{b}$ and $\mathbf{c})$ of L-box after $6 \mathrm{~s}$ for Mix 1

obstacles in the L-box apparatus (Fig. 8). The gap between the bars is $59 \mathrm{~mm}$. The number of particles used here was 59568, exactly as used in the simulation of the SCC mixes without fibres. This number of particles gives an adequate accuracy in a reasonable time. The steel fibres were treated as explained above. The number of fibres in each of these mixes was calculated from their volume fractions $(0.5 \%$ in Mix 2 and $2.5 \%$ in Mix 4) (see Table 4). The material properties of the two mixes are given in Table 4.

The time needed for Mix 2 to reach 200 and $400 \mathrm{~mm}$ from the gate was 0.5 and $1.6 \mathrm{~s}$, while for Mix 4 the time taken was 0.7 and $2 \mathrm{~s}$. It can be seen from Figs. 8 and 9 that the fibres appear to be homogenously distributed in the mix at all times during the flow in both Mix 2 and 4, exactly as observed on the same mixes in the laboratory, thus proving the effectiveness of the proposed method to simulate the filling and the passing ability of these highly viscous fluids. The 3D simulations of 
(a)

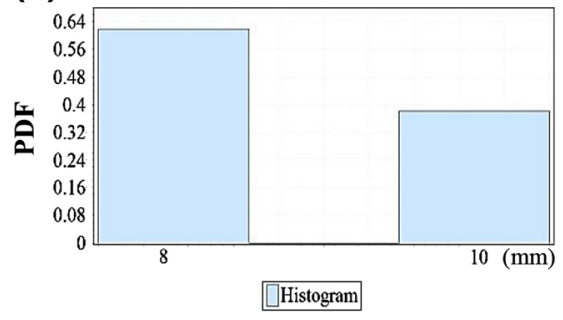

(b)

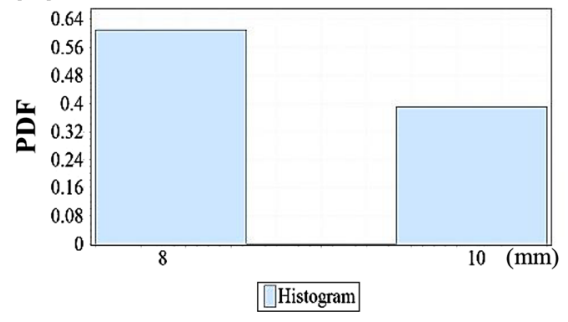

(c)

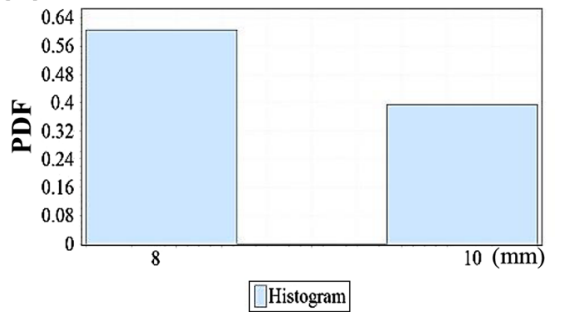

Fig. 6 Histogram of larger aggregate distribution $(\geq 8 \mathrm{~mm})$ in Mix 1 along several vertical cross-sections $(\mathbf{a}, \mathbf{b}$ and $\mathbf{c})$ after $6 \mathrm{~s}$ flow time in the L-box

Table 3 A comparison between the flow time of the SCC Mix 1 in the L-box in the $2 \mathrm{D}$ and $3 \mathrm{D}$ simulations with the laboratory test

\begin{tabular}{llll}
\hline Flow times & 2D simulation & 3D simulation & Laboratory results \\
\hline $\mathrm{t}_{200}(\mathrm{~s})$ & 0.24 & 0.30 & 0.35 \\
$\mathrm{t}_{400}(\mathrm{~s})$ & 0.60 & 0.64 & 0.76 \\
Level-off (s) & 5.90 & 6.00 & 7.23 \\
\hline
\end{tabular}

the two mixes with fibres represent an improvement over the $2 \mathrm{D}$ simulations, as is clear from Table 5 , especially at $t_{200}$. Nevertheless, based on the simulation results of both mixes, the remark made before about the inevitable delay associated with opening the gate and therefore an increase in the measured times has to be borne in mind here, too. This delay appears to be longer with mixes containing fibres (Mix 2 and 4), because of the increased pressure behind the gate and the friction between the gate and the mix, which make opening the gate in a single lift very difficult. Note that, in view of the excessive computational time needed to perform the $3 \mathrm{D}$ simulations in the L-box, the simulations were not continued until the flow levelled off in the horizontal section of the box.

\section{Fibre orientation distribution in the L-box}

The performance of fibre reinforced SCC in the hardened state is largely dictated by the alignment of fibres relative to the major tensile stresses in the cast structural element. Therefore, it is vital to know how fibres are distributed and reoriented at the end of the casting process because their locations and orientations will not change once the mix begins to harden. The procedure used below follows that used in [5] for the reorientation of fibres during the slump cone test.

Based on the simulations presented here of the fibre reorientation and distribution during the flow of mixes 2 and 4 in the L-box, it was possible to track the orientation of each individual fibre. This orientation is represented by angles $\theta_{i}$ and $\varphi_{i}$ throughout the flow of SCC, where $\theta_{i}$ spans between $0^{\circ}$ and $90^{\circ}, \varphi_{i}$ between $0^{\circ}$ and $360^{\circ}, s_{i}$ and $e_{i}$ are the start and the end of each fibre $i$, respectively (Fig. 10).

From Fig. 10 (a) it follows that

$\tan \theta_{i}=\left(\sqrt{\Delta x_{i}^{2}+\Delta y_{i}^{2}}\right) / \Delta z_{i} ; \quad \tan \varphi_{i}=\Delta x_{i} / \Delta y_{i}$

We now introduce a probability density function (PDF) of the fibre orientations $f(\theta, \varphi)$ at an arbitrary instant $t$ during the flow. The PDF satisfies $\int_{0}^{2 \pi} \int_{0}^{\frac{\pi}{2}} f(\theta, \varphi) \sin \theta d \theta d \varphi=1$. Next, we perform a statistical analysis of the fibre orientations at different times during the flow to estimate the best fit PDF. The Johnson SB distribution function [6], which has been successfully used in marine [7] and forestry [8] science, was found to fit our simulated data the best from among 55 different distribution functions tried, including the Gamma, Gaussian, uniform, log-normal, Weibull distributions, etc.

$f(x)=\frac{\delta}{\lambda \sqrt{2 \pi} z(1-z)} \exp \left(-\frac{1}{2}\left(\gamma+\delta \ln \left(\frac{z}{1-z}\right)\right)^{2}\right)$

$z=\frac{x-\xi}{\lambda}$

Equation (2), in which $x$ stands for $\theta$ or $\varphi$ represents the most probable fibre density function for each of the two uncorrelated variables $\theta, \varphi$.Here, $\lambda>0$ is a scale parameter and $\xi$ is a location parameter. $\lambda$ and $\delta$ are shape parameters of the PDF curve. The density function is skewed to the left, symmetric, or skewed to the right, if $\gamma>0, \gamma=0$, or $\gamma<0$, respectively. $\delta$ represents the standardized measure of kurtosis of the curve; a high positive value means the curve is sharp, while a small value close to 0 means a nearly flat, wide curve [5].

The Johnson SB distribution function possesses two properties that make it well suited to represent fibre orientation angles $\theta, \varphi$. Firstly, because of the lower bound $\xi$ and the upper bound $\lambda$, it can represent variables that have physical or natural constraints on their ranges. Secondly, the two parameters controlling the shape $\gamma$ and $\delta$ allow a considerable amount of flexibility to fit a broad spectrum of distributions. This is clearly seen in Figs. 11-14 which show the PDFs of 

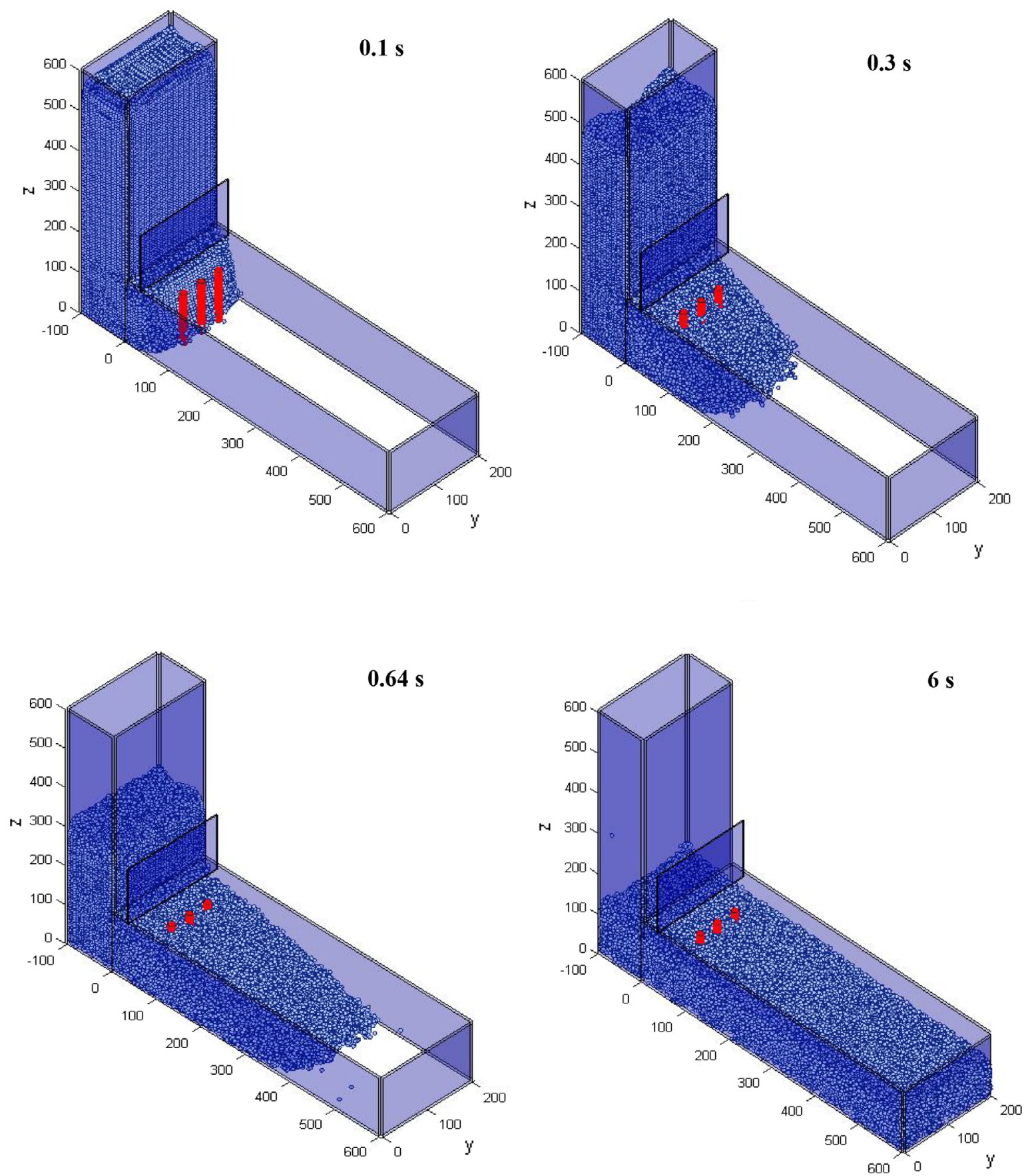

Fig. 7 The flow of SCC mix 1 as a homogeneous mass in the L-box test after $0.1 \mathrm{~s}, 0.3 \mathrm{~s}, 0.64 \mathrm{~s}$ and $6 \mathrm{~s}$

$\theta$ and $\varphi$ at various instants during the flow of Mixes 2 and 4 in the in the L-box test (up to $3 \mathrm{~s}$ flow time).

The mean values of angles $\theta$ and $\varphi$ at various instants of flow shown in Fig. 15 are calculated using Eq. (4), where $n$ is the total number of fibres

$\bar{x}=\sum_{i=1}^{n} f\left(x_{i}\right) x_{i}$

From Fig. 15, it is clear that the fibres align themselves predominantly with the principal direction of flow and the mean angle of orientation with respect to the horizontal direction of flow is only $(90-\theta) \approx 13^{\circ}$ after $3 \mathrm{~s}$ for Mix 2 and $(90-\theta) \approx 17^{\circ}$ for Mix 4 .

\section{Determination of fibre orientation in a cross-section}

In practice, the fibre orientation is often assessed by image analysis on cut sections of a hardened concrete structural element. A cylindrical fibre with diameter $d$ and length $l$ cut 

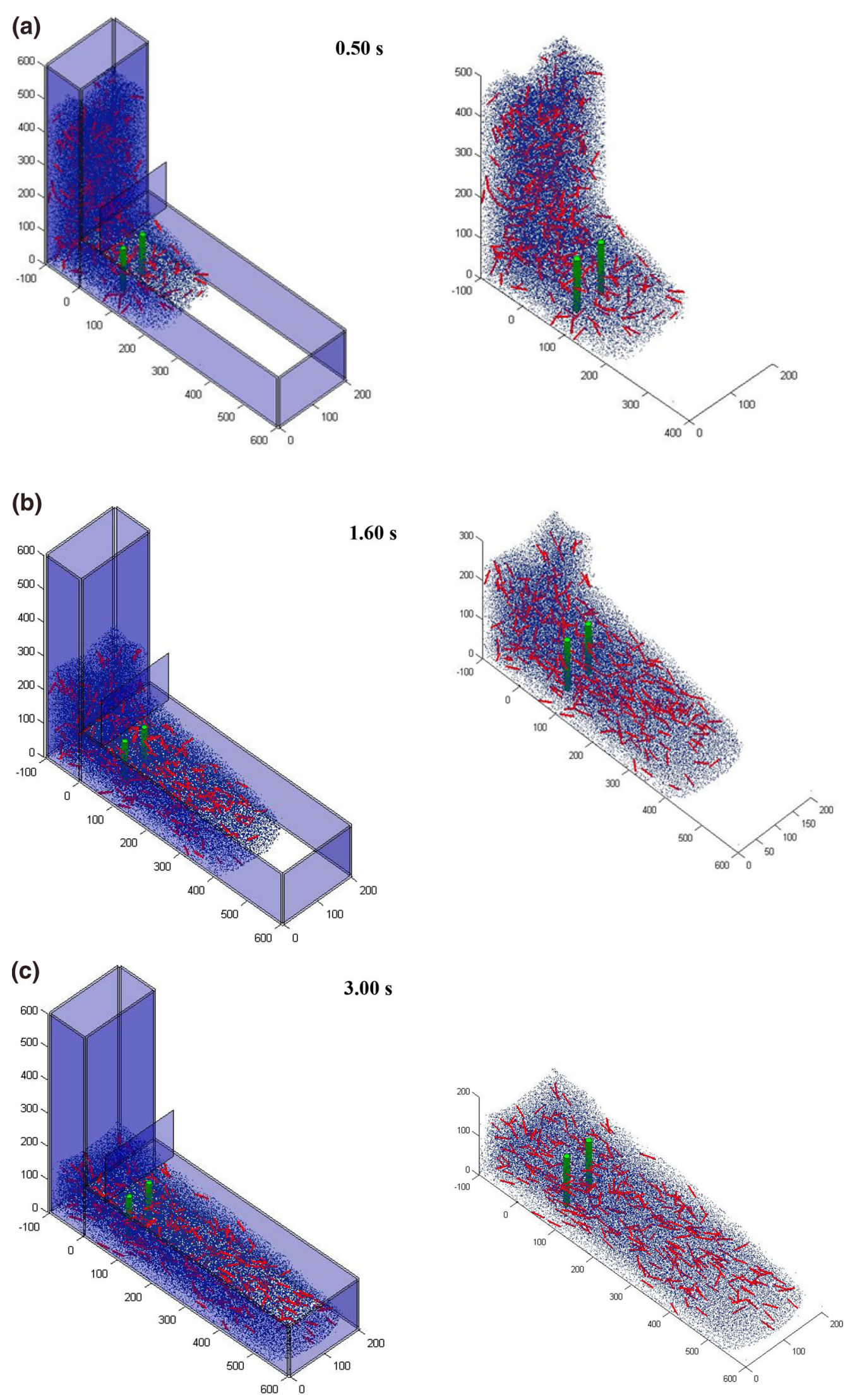

Fig. 8 The flow of Mix 2 in the L-box after a $0.50 \mathrm{~s}, \mathbf{b} 1.60 \mathrm{~s}$, and c $3.00 \mathrm{~s}$. (For clarity of presentation, the images on the right show the flow without the box) 
Table 4 Volume fraction of fibres in (Mix 2) and (Mix 4), the number of particles representing them, their material parameters and assigned volumes

\begin{tabular}{lll}
\hline & Mix 2 & Mix 4 \\
\hline Total number of particles & 59568 & 59568 \\
Volume fraction of fibres (\%) & 0.5 & 2.5 \\
Number of fibres & 298 & 1490 \\
Number of fibre end particles & 596 & 2980 \\
Assigned volume/particle $\left(\mathrm{mm}^{3}\right)$ & 340.46 & 354.66 \\
Plastic viscosity (Pa.s) & 42.10 & 54.30 \\
Yield stress (Pa) & 200 & 200 \\
\hline
\end{tabular}

by a plane will appear as a circle (with diameter $d$ ) if the plane is perpendicular to the fibre, as an ellipse if the plane is inclined to the fibre (with the aspect ratio dependent on the angle of inclination), or as a rectangle (width $d$ and length $l$ ) if it lies in the plane.

By cutting the L-box by a vertical plane at any time during the flow, we can calculate exactly the number and crosssectional shapes of the fibres cut by this plane. For this, we need to calculate the centre of intersection between a cylindrical fibre and the cutting plane, say the vertical plane $y z$ at $x=x_{c i}$. Let us denote the coordinates of the centre of intersection as $\left(x_{c i}, y_{c i}, z_{c i}\right)$. These coordinates and are calculated from the coordinates of the ends of a fibre (Fig. 10 (b)) and the plane $x=x_{c i}$.

$$
\begin{aligned}
& y_{c i}=y_{s i}+\left(\left(y_{e i}-y_{s i}\right)\left(x_{c i}-x_{s i}\right) /\left(x_{e i}-x_{s i}\right)\right) ; \\
& z_{c i}=z_{s i}+\left(\left(z_{e i}-z_{s i}\right)\left(x_{c i}-x_{s i}\right) /\left(x_{e i}-x_{s i}\right)\right)
\end{aligned}
$$

From Fig. 10(b) it follows that

$$
\tan \theta_{i}=\left(\sqrt{\Delta y_{i}^{2}+\Delta z_{i}^{2}}\right) / \Delta x_{i} ; \quad \tan \varphi_{i}=\Delta y_{i} / \Delta z_{i}
$$

The shape of the cross-section of the fibre cut by a vertical plane is decided as follows:

- It is rectangular (with width $=d$ and length $=l$ ) if $x_{s i}=x_{e i}$, as both ends of the fibre will appear in the plane $y z$. In this case, $\theta_{i}=90^{\circ}$;

- It is circular (with diameter $=d$ ) if $x_{s i}$ is not equal to $x_{e i}$ and $\theta_{i}=0^{\circ}$ (Fig. 10 (b));

- It is elliptical otherwise, i.e. if $x_{s i}$ is not equal to $x_{e i}$ and $\theta_{i}$ is not equal to $0^{\circ}$.

- The semi major $\left(a_{i}\right)$ and semi minor $\left(b_{i}\right)$ axes of the ellipse can be calculated once the angle $\theta_{i}$ (the angle between the fibre and the axis normal to the cut plane) is known from Eq. (6); it spans between $0^{\circ}$ and $90^{\circ}$. These are

$$
a_{i}=r / \cos \theta_{i} ; b_{i}=r
$$

where the fibre radius $r=d / 2=0.275 \mathrm{~mm}$.

At this stage all the required geometrical data for the ellipses are known, namely the centre $c_{i}\left(x_{c i}, y_{c i}, z_{c i}\right)$, the semi-major $a_{i}$, and the semi-minor $b_{i}$ axes.

The major axis of the ellipse may also be rotated by an angle $\varphi_{i}$ (spanning between 0 and 180 degrees with respect to $x$-axis as illustrated in Fig. 10(b) which can be calculated from Eq. (6).

Two vertical sections will be taken; the first one at 200 $\mathrm{mm}$ from the vertical leg of the L-box and the second at 300 $\mathrm{mm}$.

Figures 16-19 illustrate how the steel fibres are oriented in a vertical section of the self-compacting concrete mixes (Mix 2 and Mix 4) parallel to the $y z$ plane at various times during flow in the horizontal part of the L-box flow. For clarity of presentation, the sizes of the ellipses, circles, and rectangles are exaggerated several times compared to the cross section dimensions of the L-box. Note that the coordinates of the particles representing the ends of the fibres are continuously monitored throughout the simulation from which the crosssectional shapes of the fibres cut by the vertical sections are calculated as described above.

It should be mentioned that the end particles representing the fibres are randomly distributed, but as the distance between these particles is fixed and equal to the fibre length, a number of degrees of freedom are suppressed. Referring to Table 4, this means that 298 degrees of freedom out of a possible 178704 are suppressed in Mix 2 and 1490 in Mix 4.

It can be seen from Figs. 16-19 that the number of fibres cut by the vertical section (plane $y z$ ) decreases as the flow time increases and the surface area of cut section reduces. It should be mentioned that for the L-box, when the flow stops, the surface area of all cut sections should be nearly the same, therefore the number of fibres should be same in all the vertical cross sections. These results are not available because of the long computational time needed for the flow to stop.

For a random distribution of fibres in a volume, the number of fibres lying in section of a certain area or cut by it can be calculated exactly using the theory of geometric probability, or more precisely from the solution of the so-called Buffon problem $[9,10]$. The solution is

$l \frac{E(h)}{E(c)}=4 \frac{\sum \text { volumes }}{\text { surfaceareas }}$

The solution is obtained by dividing the volume of the container into smaller cells, say $40 \mathrm{~mm}$ cubes and then calculating the number of fibres of equal length that either fall into interior of the cells or are cut by one of its surfaces. Here, $E(c)$ is the number of fibres of length $l$ that are cut by one of the surfaces of the cells, and $E(h)$ is the number of times each of the two ends of the fibres fall in the interior of the cells. 

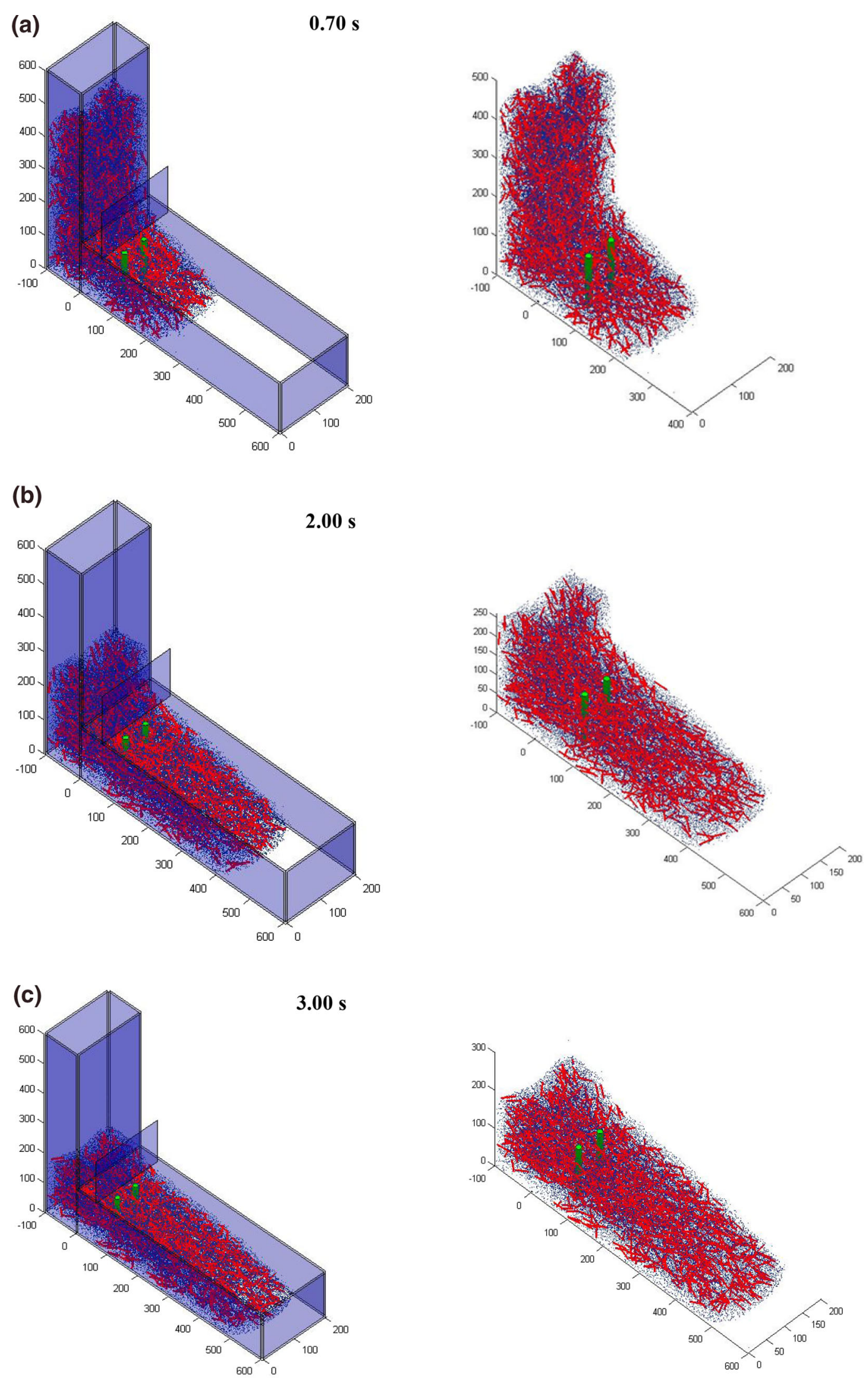

Fig. 9 The flow of Mix 4 in the L-box test after a 0.70 s, b 2.00 s, and c $3.00 \mathrm{~s}$. (For clarity of presentation, the images on the right show the flow without the box) 
Table 5 A comparison between the flow times of the SCC Mixes 2 and 4 in the 2D and 3D simulations with the laboratory test

\begin{tabular}{|c|c|c|c|c|c|c|}
\hline \multirow[t]{2}{*}{ Flow times } & \multicolumn{2}{|c|}{ 2D simulation } & \multicolumn{2}{|c|}{ 3D simulation } & \multicolumn{2}{|c|}{ Laboratory results } \\
\hline & Mix 2 & Mix 4 & Mix 2 & Mix 4 & Mix 2 & Mix 4 \\
\hline $\mathrm{t}_{200}(\mathrm{~s})$ & 0.40 & 0.50 & 0.50 & 0.70 & 1.88 & 1.90 \\
\hline $\mathrm{t}_{400}(\mathrm{~s})$ & 1.60 & 2.00 & 1.60 & 2.00 & 5.10 & 5.60 \\
\hline Level-off (s) & 38.00 & 50.00 & - & - & 38.00 & 54.00 \\
\hline
\end{tabular}

(a)

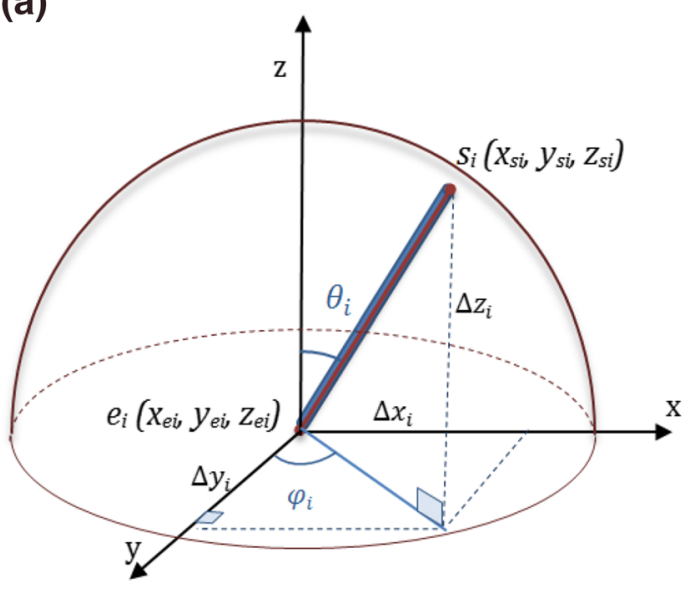

(b)

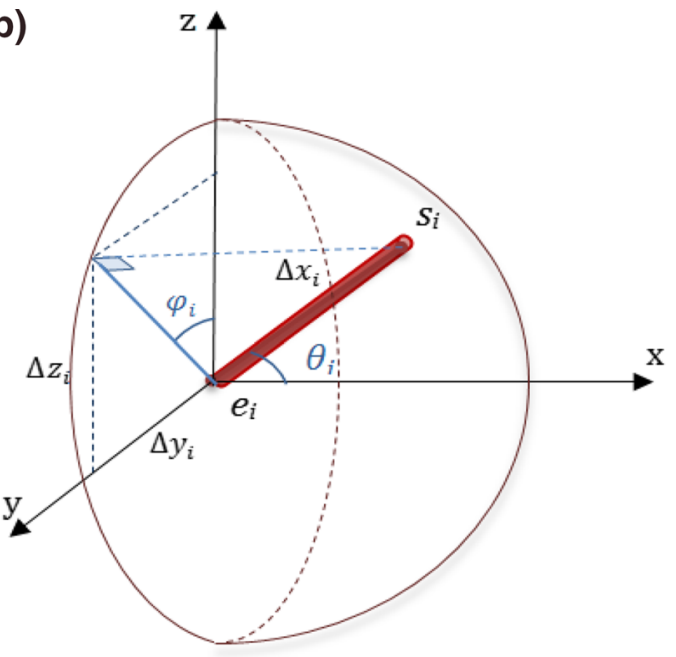

Fig. 10 Fibre orientation in three dimensions: a with respect to a horizontal plane parallel to $x y$, and $\mathbf{b}$ with respect to a vertical plane parallel to $y z$

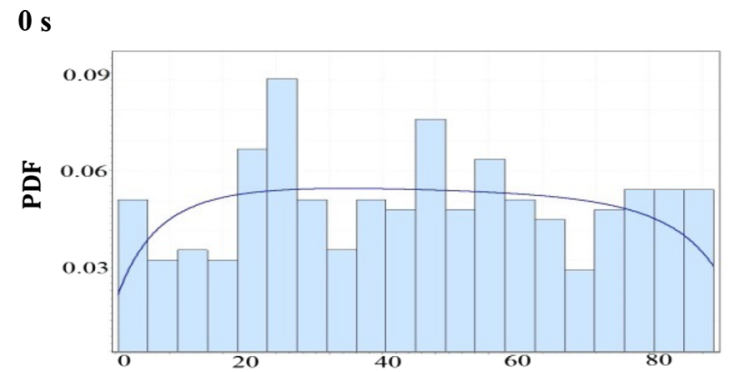

Angle $\theta$ (degree)

$1.8 \mathrm{~s}$

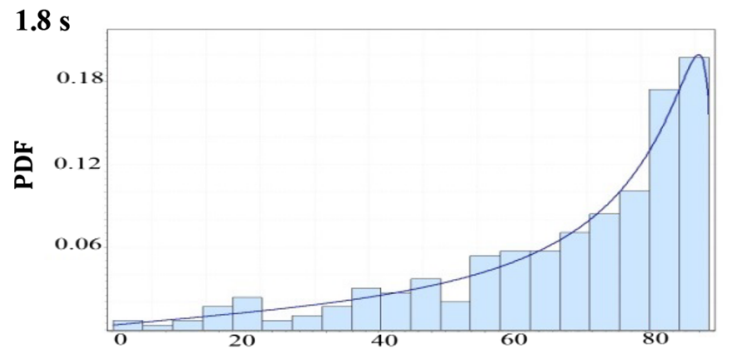

Angle $\theta$ (degree)

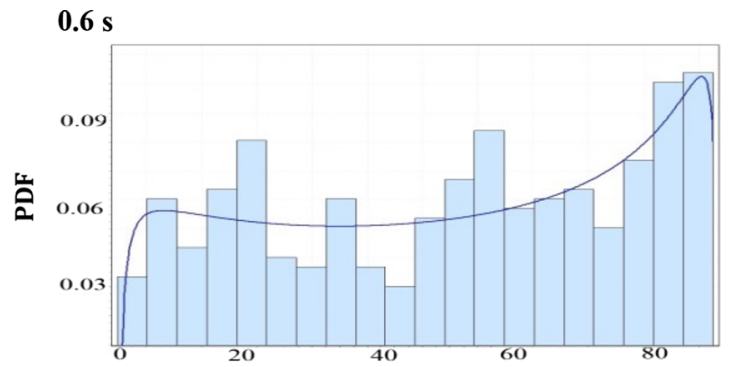

Angle $\boldsymbol{\theta}$ (degree)

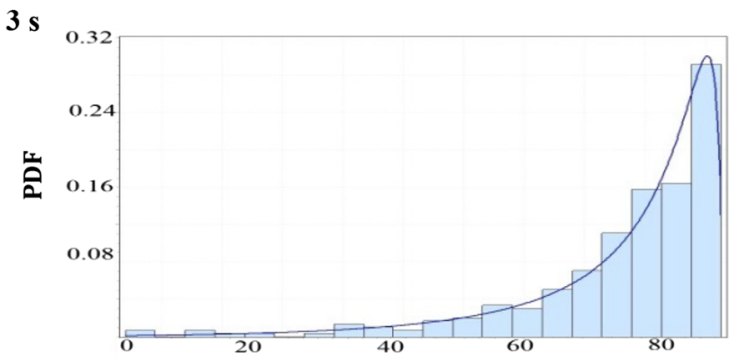

Angle $\boldsymbol{\theta}$ (degree)

Histogram -Johnson SB

Fig. 11 Probability density function $f(\theta)$ of Mix 2 at several instants during the flow in the L-box 

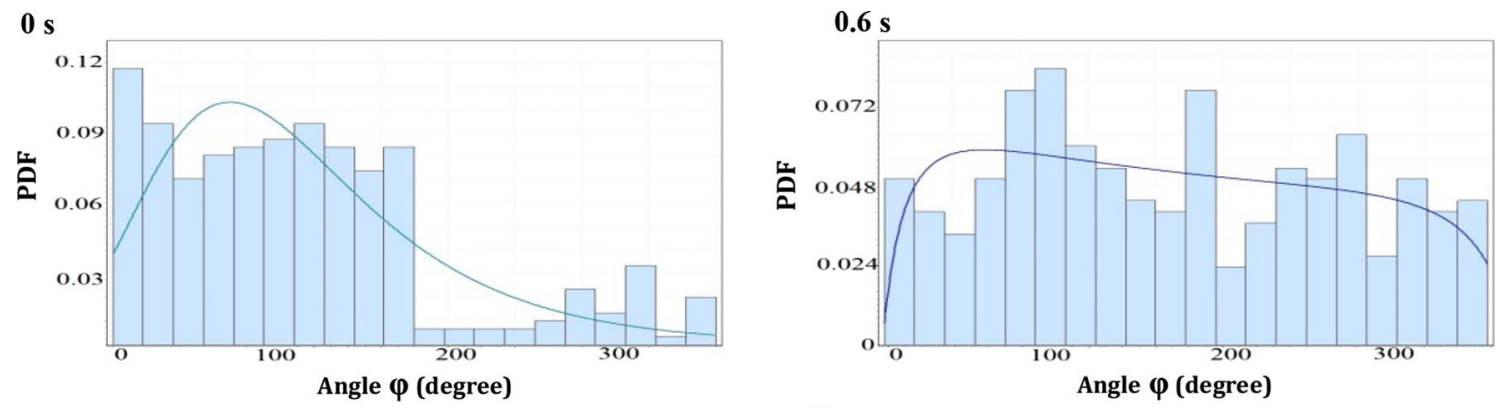

$1.8 \mathrm{~s}$

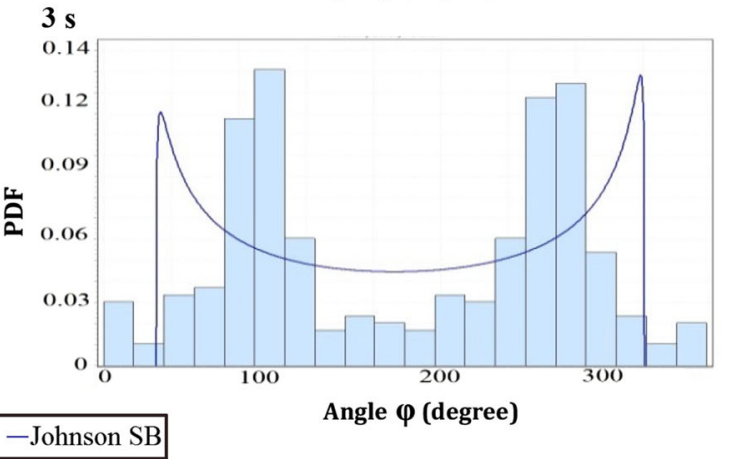

Fig. 12 Probability density function $f(\varphi)$ of Mix 2 at several instants during the flow in the L-box

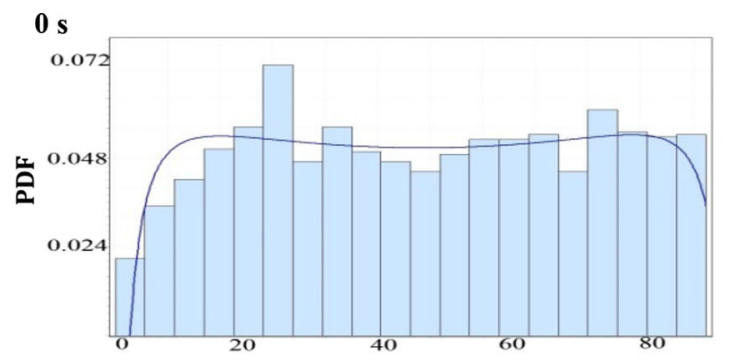

Angle $\boldsymbol{\theta}$ (degree)

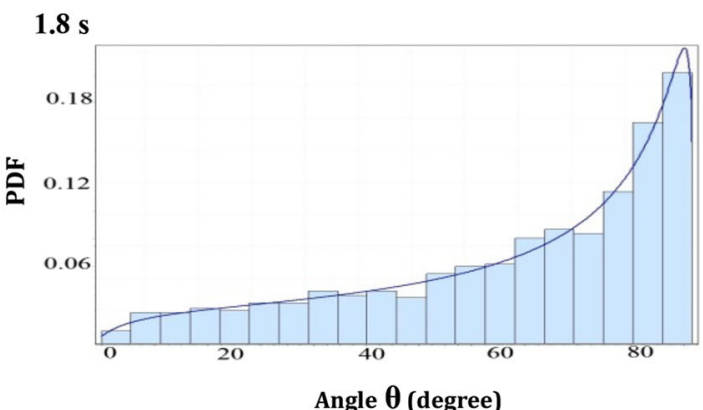

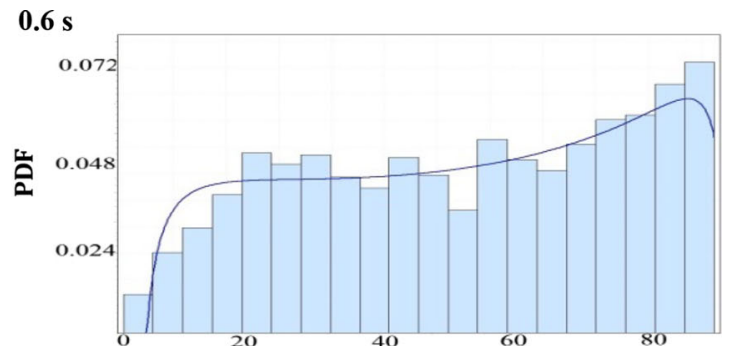

Angle $\boldsymbol{\theta}$ (degree)

$3 \mathrm{~s}$

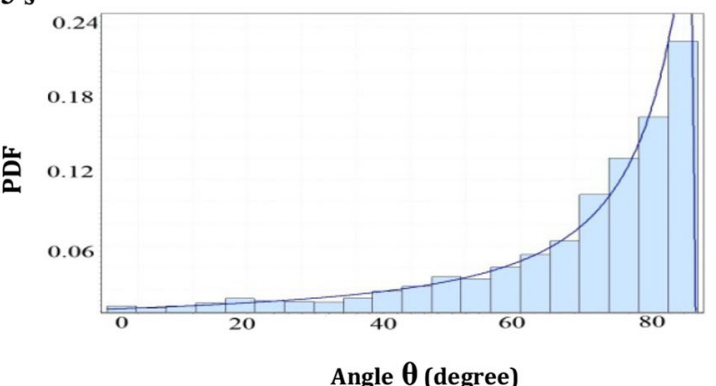

$\square$ Histogram -Johnson SB

Fig. 13 Probability density function $f(\theta)$ of Mix 4 at several instants during the flow in the L-box

By using the theory of geometric probability for Mix 4 containing $2.5 \%$ volume fraction of $30 \mathrm{~mm}$ long steel fibres with $0.55 \mathrm{~mm}$ diameter, the total number of fibres in the volume of the concrete in the L-box is

$N_{f}=\frac{V_{c}}{V_{f}} * 2.5 \%=45250$ where $V_{c}$ is the volume of concrete in the L-box and $V_{f}$ is the volume of each fibre. By dividing the concrete volume into $40 \mathrm{~mm}$ cubical cells (there are nearly 202 such cells) we have from Eq. (8) that

$\frac{E(h)}{E(c)}=\frac{8}{9}$ 

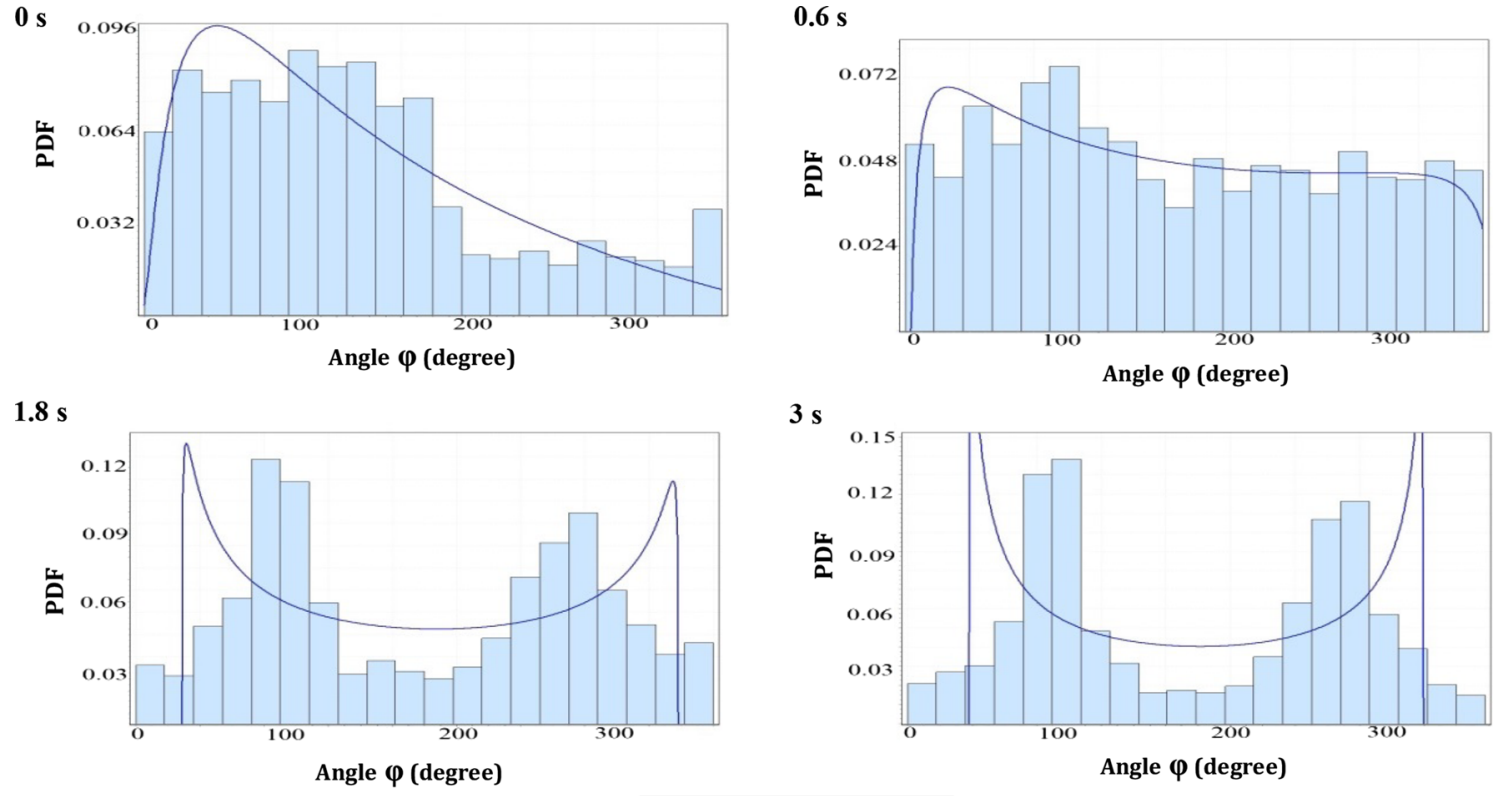

Fig. 14 Probability density function $f(\varphi)$ of Mix 4 at several instants during the flow in the L-box

Fig. 15 The mean values of angles $\theta$ and $\varphi$ for Mix 2 (a) and Mix 4 (b) in L-box test
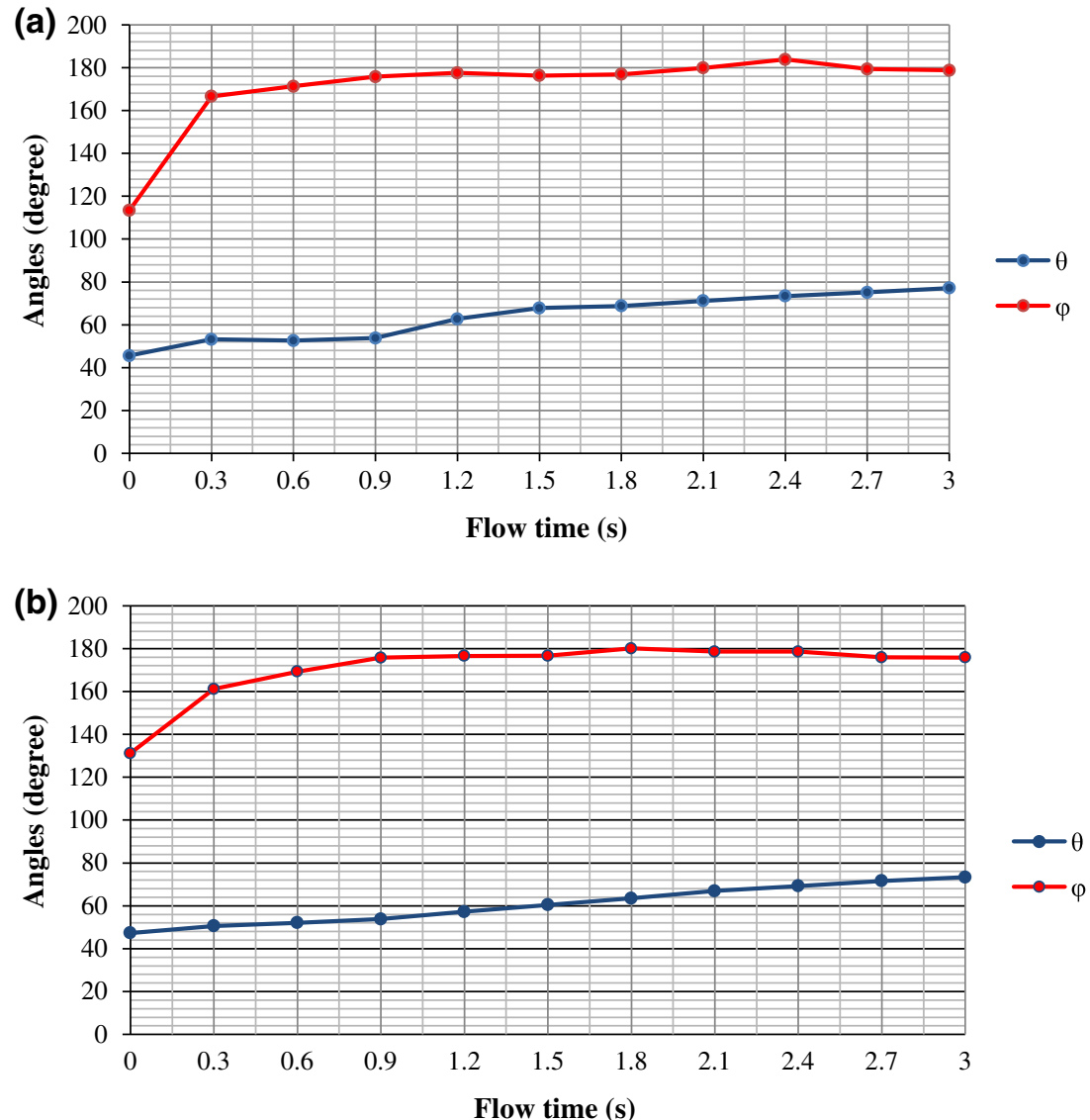

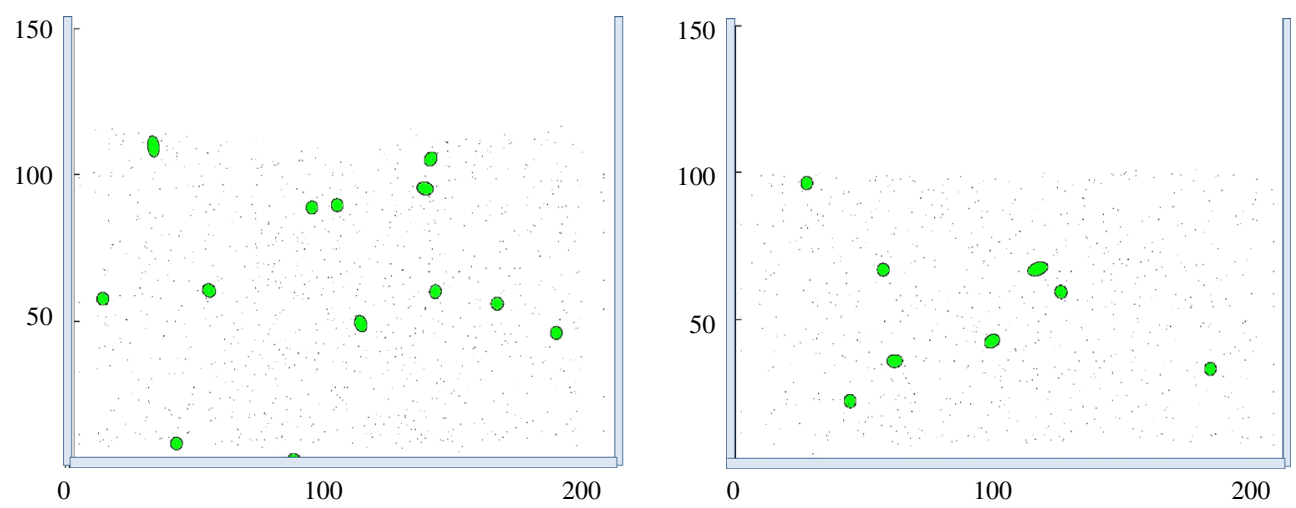

Fig. 16 Vertical cross sections at $200 \mathrm{~mm}$ (left) and $300 \mathrm{~mm}$ (right) after $2 \mathrm{~s}$ in L-box (Mix 2)
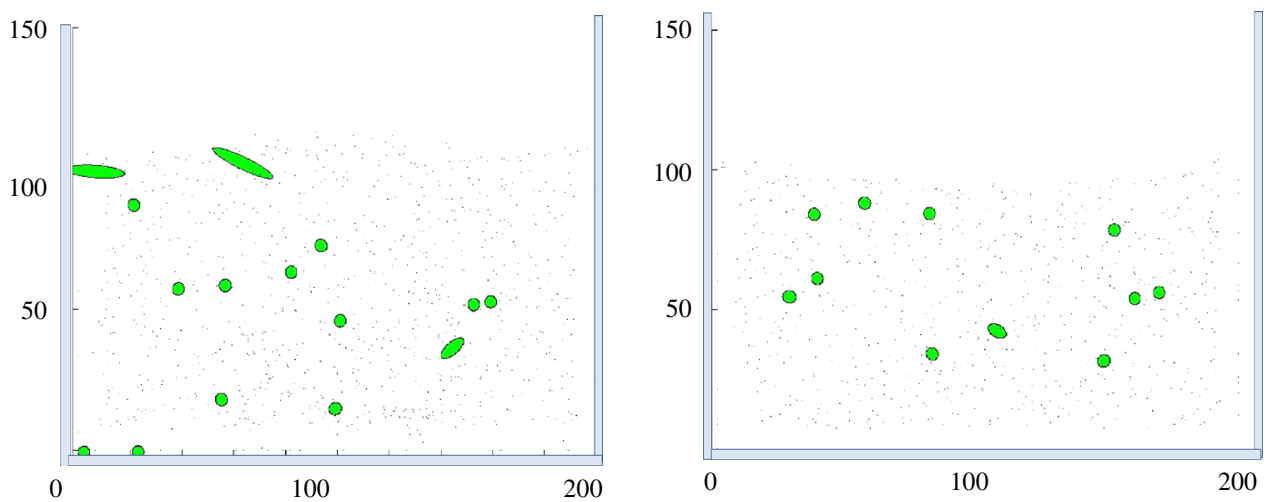

Fig. 17 Vertical cross sections at $200 \mathrm{~mm}$ (left) and $300 \mathrm{~mm}$ (right) after $3 \mathrm{~s}$ in L-box (Mix 2)
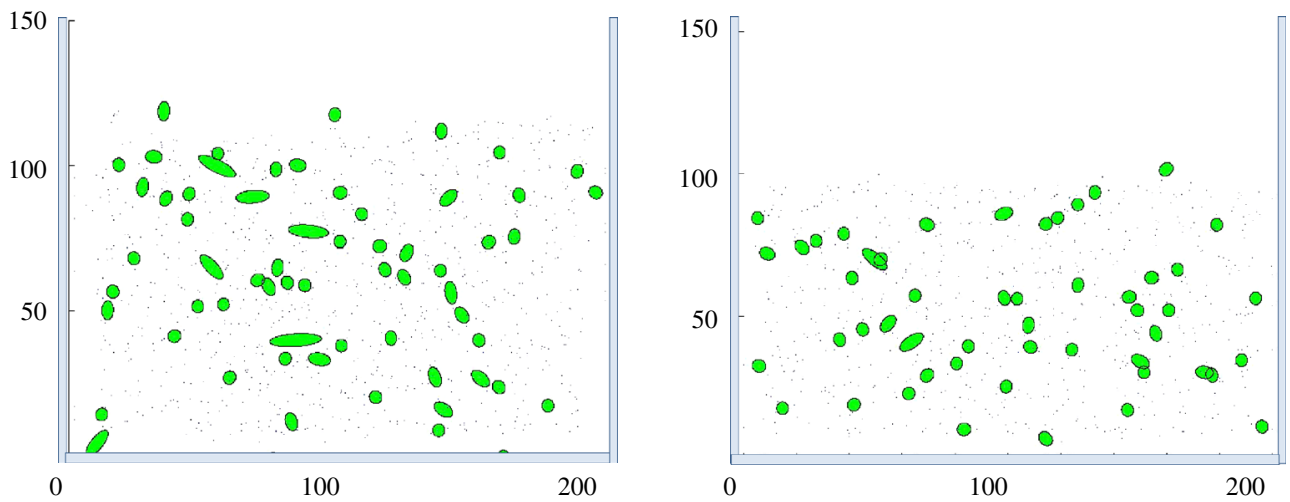

Fig. 18 Vertical cross sections at $200 \mathrm{~mm}$ (left) and $300 \mathrm{~mm}$ (right) in the L-box after $2 \mathrm{~s}$ (Mix 4)

The total number of fibres in the interior of each cell or cut by one of its surfaces is

$E(c)+E(h)=\frac{V_{\text {cube }}}{V_{f}} * 2.5 \%=224$,

hence it follows that the number of fibres cut by $40 \times 40 \mathrm{~mm}^{2}$ side of cube is $E(c)=118$. The vertical section of the Lbox at $200 \mathrm{~mm}$ (Fig. 18) has a surface area equal to 25400 $\mathrm{mm}^{2}$, so that the total number of fibres cut by this section is $\frac{118}{40 * 40} * 25400=1873$ fibres out of a total of 45250 fibres in the L-box (Eq. (9)). The number of fibres that should theoretically be cut by the vertical cross-section of the L-box at $200 \mathrm{~mm}$ after $2 \mathrm{~s}$ flow time is $1490 * 1873 / 45250=62$ fibres. Table 6 compares the numbers of fibres in a vertical crosssection based on the 3D simulations with the Buffon problem solution. The small difference between the theoretical and simulated numbers is because of the pseudo-random nature of the fibre distribution in the simulations. The distribution is pseudo-random because some of the degrees of freedom of the fibre end particles are suppressed, as explained above. 

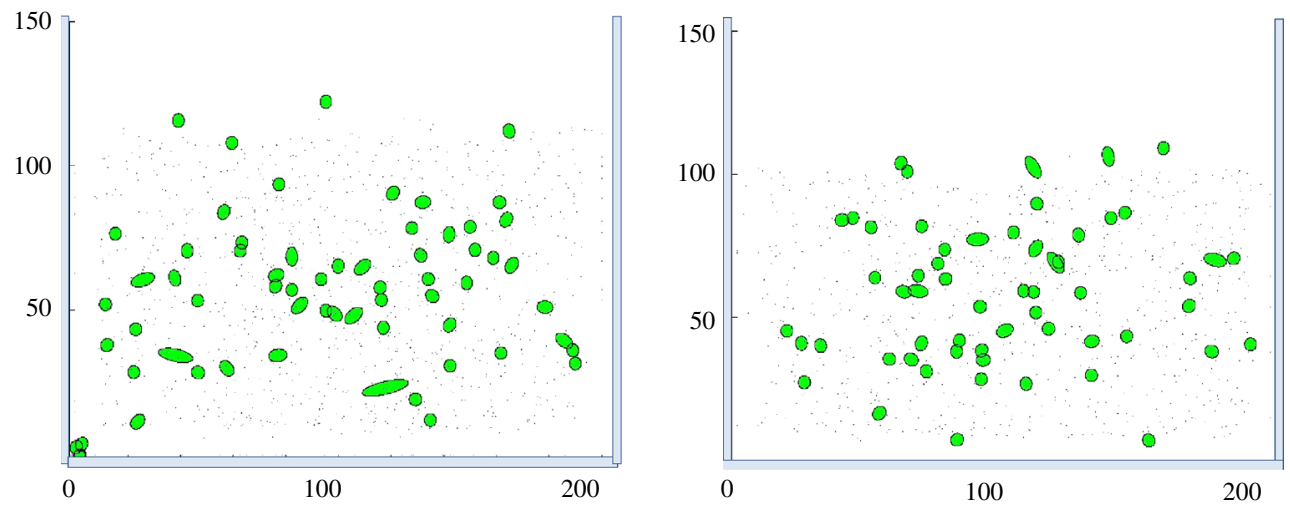

Fig. 19 Vertical cross sections at $200 \mathrm{~mm}$ (left) and $300 \mathrm{~mm}$ (right) in the L-box after $3 \mathrm{~s} \mathrm{(Mix} \mathrm{4)}$

Table 6 Number of fibres in a vertical cross-section of (Mix 2) and (Mix 4) in L-box test based on the 3D simulation and Buffon problem

\begin{tabular}{llrrrr}
\hline & Flow time (s) & \multicolumn{2}{l}{$\begin{array}{l}\text { No. of fibres } \\
\text { in the simulation }\end{array}$} & \multicolumn{2}{c}{$\begin{array}{l}\text { No. of fibres based on } \\
\text { Buffon problem }\end{array}$} \\
\hline Section $(\mathrm{mm})$ & & 200 & 300 & 200 & 300 \\
Mix 2 & 2 & 13 & 8 & 13 & 10 \\
& 3 & 16 & 11 & 13 & 11 \\
Mix 4 & 2 & 63 & 51 & 62 & 49 \\
& 3 & 64 & 58 & 62 & 56 \\
\hline
\end{tabular}

\section{Fibre orientation factor}

A FOF $\eta(0 \leq \eta \leq 1)$ is used to assess the orientation state of fibres in a given cross-section. If this orientation factor can be controlled and predicted effectively, then the partial safety factor reflecting the fibre orientations can be chosen close to unity thus allowing the superior properties of fibre reinforced self-compacting concrete to be exploited to the full extent [11]. It was first proposed by Soroushian and Lee [12] as the ratio of the number of fibres actually counted in a given cross-section $(N)$ to the average theoretical number of fibres in such a cross-section $\left(N_{t h}\right)$, assuming that all the fibres are perpendicular to the cut surface. The theoretical number of fibres was chosen equal to the area of all fibres in the cross section $\left(A_{c} * V_{f}\right)$ divided by the area of one fibre $\left(A_{f}\right)$, where $\left(A_{c}\right)$ is the area of the cross-section and $V_{f}$ is the volume fraction of fibres [11]. The lower this factor, the larger is the average deviation of the fibres from the perpendicular direction to the cut surface.

There are two drawbacks of this definition of the fibre orientation factor. Firstly, the $N_{t h}$ calculated in the prescribed approximate manner overestimates the actual theoretical number of fibres in a cross section. This number can however be exactly calculated using the theory of geometric probability described above [9]. Secondly, $N$ does not reflect the actual inclination of the fibres relative to the section, and thus does not reflect the effectiveness of a fibre; a fibre is most effective if it is perpendicular to the cross-section; it is completely ineffective if it lies in the section; a fibre inclined to the section is partially effective depending on its angle of inclination.

Therefore, a new orientation factor is introduced here. It is defined as the ratio of the projected length along the normal to the cut plane ( $x$-axis for vertical plane $y z$ ) to the actual length $l$ of the fibre. This ratio is nothing but the cosine of the angle between the fibre and the $x$-axis (Fig. 10(b)). This definition is consistent with the fibre probability density function introduced above. The orientation factor in the vertical cut plane is:

$\bar{\eta}=\frac{1}{n} \sum_{i=1}^{n} \cos \theta_{i}=\frac{1}{n} \sum_{i=1}^{n} \frac{r}{a_{i}}$

It is clear from Figs. 20 and 21 that the fibres tend to reorient themselves with the direction of flow, irrespective of the fibre volume fraction; corresponding to a fibre orientation factor of about 0.90 and 0.92 , respectively.

By comparison, according to the definition of Soroushian and Lee [12] the FOF for Mix 2 and Mix 4 after 2 s flow of the L-box at $200 \mathrm{~mm}$ cross-section would be $(11 / 16)=0.69$ and $(13 / 19)=0.68$, respectively. The corresponding values after 3 s flow of the L-box at $300 \mathrm{~mm}$ cross-section for Mix 2 and Mix 4 are $(56 / 81)=0.69$ and $(62 / 90)=0.68$, respectively. In other words, the FOF hardly changes with time, i.e. the fibres do not reorient with the principal flow direction at all which is clearly unexpected and counterintuitive in SCC mixes. In calculating the FOF according to Soroushian and Lee [12] 


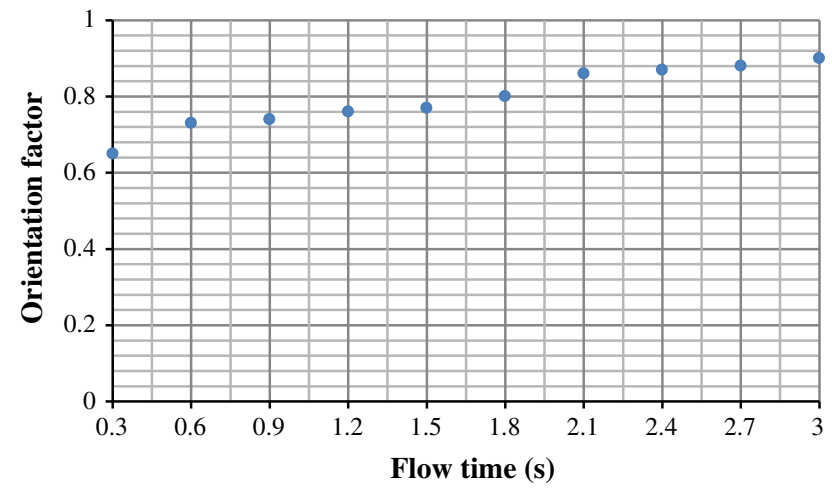

Fig. 20 Fibre orientation factor in a vertical cut section at $100 \mathrm{~mm}$ of Mix 2 in the L-box

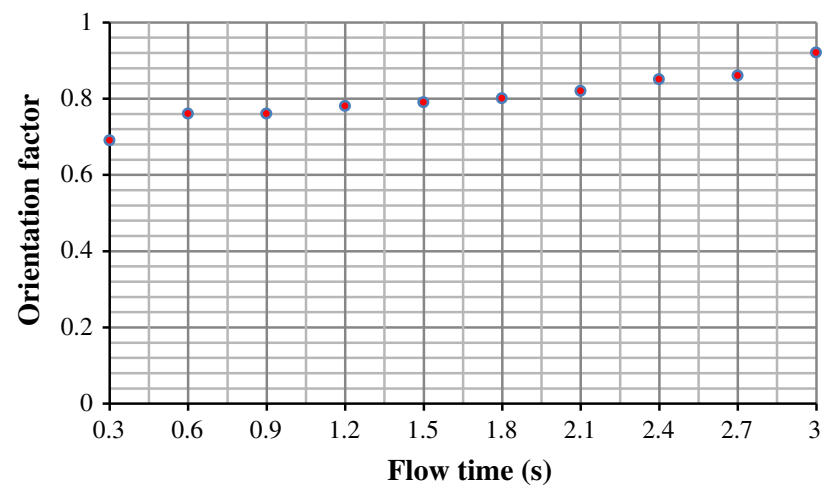

Fig. 21 Fibre orientation factor in a vertical cut section at $100 \mathrm{~mm}$ of Mix 4 in the L-box

we have used $N_{t h}$, as explained above and $N$ according to the solution of the Buffon problem (Table 6).

Finally, it should be mentioned that in practice it may not be possible or practicable to perform the full threedimensional simulation of the flow of fibre-reinforced SCC into the formwork and thus to determine the mean angle of inclination of fibres $\theta$ needed for the calculation of the FOF (first equality in Eq. (12)). In such instances, it is necessary to count all the fibres in the cut section, noting the fibres with circular sections, measuring the major axis of all fibres with elliptical sections, and using the second equality in Eq. (12) to calculate the FOF. Note that the fibres that lie in the plane along which the specimen is cut (i.e. $\theta_{i}=90^{\circ}$ ), and which are thus likely to come loose and fall during cutting, need not be taken into account in any case because they make a negligible contribution to the fibre orientation factor.

\section{Concluding remarks}

A Lagrangian Smooth Particle Hydrodynamic (SPH) method has been used to simulate the flow of self-compacting high and ultra-high performance concrete with and without short steel fibres in the L-box 3-dimensional configuration. A suitable Bingham model [4] has been coupled with the Lagrangian momentum and continuity equations to model the flow. The mix characteristics of the SCC mix have been fully incorporated implicitly through the plastic viscosity, which has been calculated using the micromechanical model described in [13].

The simulation of SCC mixes without fibres followed the distribution of aggregates of different sizes $(8$ and $10 \mathrm{~mm})$ throughout the flow, while those of the SCC mixes with fibres focused on the distribution of fibres and their orientations during the flow.

In the L-box simulations, although the simulation results were able to predict accurately the distribution of fibres and there orientations during the flow, there was an unavoidable delay in the flow time measured in the laboratory due to the difficulty of opening the gate in a single lift. This delay should be considered when analysing the filling ability of L-box test.

The orientation and the distribution of the steel fibres have been monitored throughout the flow and the change in orientation has been described by the Johnson SB distribution function. It is shown that the fibres tend to align themselves with the principal direction of flow but remain mostly randomly distributed perpendicular to this direction. The reorientation of the fibres during the flow has been used to estimate the FOF in a cross section perpendicular to the principal direction of flow. This estimation procedure involves the number of fibres cut by the section and their inclination to the cutting plane. It is shown that the number of fibres cut by the plane as monitored in the SPH simulation is in excellent agreement with the theoretically expected value, thus confirming the validity of the definition of FOF introduced above.

\section{References}

1. Deeb R, Kulasegaram S, Karihaloo BL (2014) 3D modelling of the flow of self-compacting concrete with or without steel fibres. Part I: slump flow test, Comput Particle Mech (this issue).

2. Kulasegaram S, Karihaloo BL (2013) Fibre-reinforced, selfcompacting concrete flow modelled by SPH. Proc ICE Eng Comput Mech 166(EM1):22-31

3. Tviksta, LG (2000) Guidlines- Task 9-End product. Brite EuRam Proposal No. BE96-3801.

4. Kulasegaram S, Karihaloo BL, Ghanbari A (2011) Modelling the flow of self-compacting concrete. Intern J Numer Anal methods Geomech 35:713-726

5. Deeb R, Kulasegaram S, Karihaloo BL (2014) Reorientation of short steel fibres during the flow of self-compacting concrete mix and determination of the fibre orientation factor. Cement Concrete Res 56:112-120

6. Johnson N (1949) Systems of frequency curves generated by methods of translation. Biometrika 36:149-176

7. Fonseca TF, Marques CP, Parresol B (2009) Describing Maritime Pine Diameter Distributions with Johnson's SB Distribution Using a New All-Parameter Recovery Approach. Soc Am For 55:367-373 
8. Rennolls K, Wang M (2005) A new parameterization of Johnson's SB distribution with application to fitting forest tree diameter data. Can J For Res 35:575-579

9. Solomon H (1978) Geometric probability, society for individual and applied mathematics. SIAM, USA

10. Crofton MW (1885) Probability, Encyclopedia Brittanica, $9^{\text {th }} \mathrm{ed}$., 19:768-788.

11. Martinie L, Roussel N (2011) Simple tools for fiber orientation prediction in industrial practice. Cement Concrete Res 41:9931000

12. Soroushian P, Lee CD (1990) Distribution and orientation of fibres in steel fibre reinforced concrete. ACI Mater J 87:433-441
13. Ghanbari A, Karihaloo BL (2009) Prediction of the plastic viscosity of self-compacting steel fibre reinforced concrete. Cement Concrete Res 39:1209-1216

14. Deeb R, Ghanbari A, Karihaloo BL (2012) Development of selfcompacting high and ultra- high performance concretes with and without steel fibres. Cement Concrete Compos 34:185-190

15. Deeb R, Karihaloo BL (2013) Mix proportioning of selfcompacting normal and high strength concretes. Mag Concrete Res 65:546-556 